\title{
Results from the 35-ton Liquid-argon Prototype using Cosmic Rays and Lessons Learned
}

\author{
Michael Wallbank ${ }^{* \dagger}$ \\ University of Sheffield \\ E-mail: wallbank@fnal.gov
}

\begin{abstract}
Liquid argon time projection chambers (LArTPCs) provide a robust and elegant method for measuring the properties of neutrino interactions above a few tens of $\mathrm{MeV}$ by providing 3D event imaging with excellent spatial resolution. LArTPCs have been chosen by the Deep Underground Neutrino Experiment (DUNE) as the far detector technology and it will play an essential role in the studies of neutrino mass ordering and $\mathrm{CP}$ violation as well as searches for proton decays and supernova neutrinos. The 35-ton prototype, located at Fermilab, featured many advanced technologies being considered by the DUNE far detector, such as the membrane cryostat, cold electronics, anode plane assembly (APA) with wrapped wires, silicon photomultipliers to readout scintillation photons, scintillator counters to trigger cosmic ray muons. The high quality data in the 35-ton LArTPC are important to the understanding of many physics effects such as the attenuation caused by impurities, diffusion, space charge, and calorimetric response of muons, protons and neutral pions.
\end{abstract}

EPS-HEP 2017, European Physical Society conference on High Energy Physics 5-12 July 2017

Venice, Italy

\footnotetext{
* Speaker.

†n behalf of the DUNE and 35-ton collaborations.
} 


\section{The DUNE Experiment}

The Deep Underground Neutrino Experiment (DUNE) [1] is a future long-baseline neutrino oscillation experiment with a rich program in neutrinos, nucleon decay and astroparticle physics. It will utilise the LBNF muon neutrino beam at Fermi National Accelerator Laboratory (FNAL) and a large underground detector based at the Sanford Underground Research Facility (SURF), providing a baseline of $1300 \mathrm{~km}$. A high-precision near detector will facilitate searches for neutrino oscillations at the far detector and also allow detailed measurements of neutrino interactions. The far detector will consist of 40 kton fiducial liquid argon (LAr) built in four staged 10 kton modules.

\subsection{The LArTPC Concept}

A LArTPC (Liquid-Argon Time Projection Chamber) utilises a volume of LAr as both the target and detector medium. When a charged particle passes through, the resulting ionisation electrons drift in an electric field (enabled by Anode Planes and Cathode Planes) towards the positively biased anode wires. In DUNE, three planes of such wires are used with differing orientations; combining information from the views enables 3D reconstruction. There are two induction planes, inducing charge whilst remaining transparent to the drift electrons, and one collection plane, on which the field lines end. A photon detection system (PDS) is additionally used to trigger on scintillation light and provide an absolute event time for the interaction. The charge observed at the anode planes is related to the purity of the LAr; electronegative impurities will capture drift electrons and result in a poorer signal. This gives rise to the concept of 'electron lifetime' to represent the inherent purity of the liquid; the DUNE requirement is $3 \mathrm{~ms}$.

\subsection{The DUNE Single-Phase Detector Design}

The DUNE single-phase detector design is illustrated in Figure 1. The field is provided by Anode and Cathode Plane Assemblies (APAs and CPAs), which are suspended and tiled to increase the active volume. A single module contains $2 \times 3 \times 22$ APAs. The collection wires are vertical and read out a single side of an APA but induction wires wrapped at angles $\pm 37^{\circ}$ to the vertical, enabling multiple drifts regions to be read out simultaneously using the same number of channels and allowing the APAs to be positioned close together. The readout consists of cold electronics (signal shaping, preamplifiers and digitisers) and is placed at the top of a top APA and at the bottom of a bottom APA. The PDS is integrated into the APAs as wavelength-shifting enhanced lightguides with SiPM readout. A grounded mesh exists at the centre of each APA to ensure a uniform field and a fourth, non-active, wire plane is incorporated on the outside to shield charge from the active wires. A high voltage of $-180 \mathrm{kV}$ on the cathode provides a drift field of $500 \mathrm{~V} / \mathrm{cm}$.

This novel design will allow DUNE to maximise its physics potential but requires prototyping to ensure it will perform as expected. The 35-ton prototype included a small-scale DUNE-style detector to test as many of the design features as possible.

\section{The 35-ton Prototype}

The 35-ton prototype [3] was constructed to demonstrate the use of membrane cryostat technology for a large experiment such as DUNE. It was the first use of membrane cryostat for scien- 


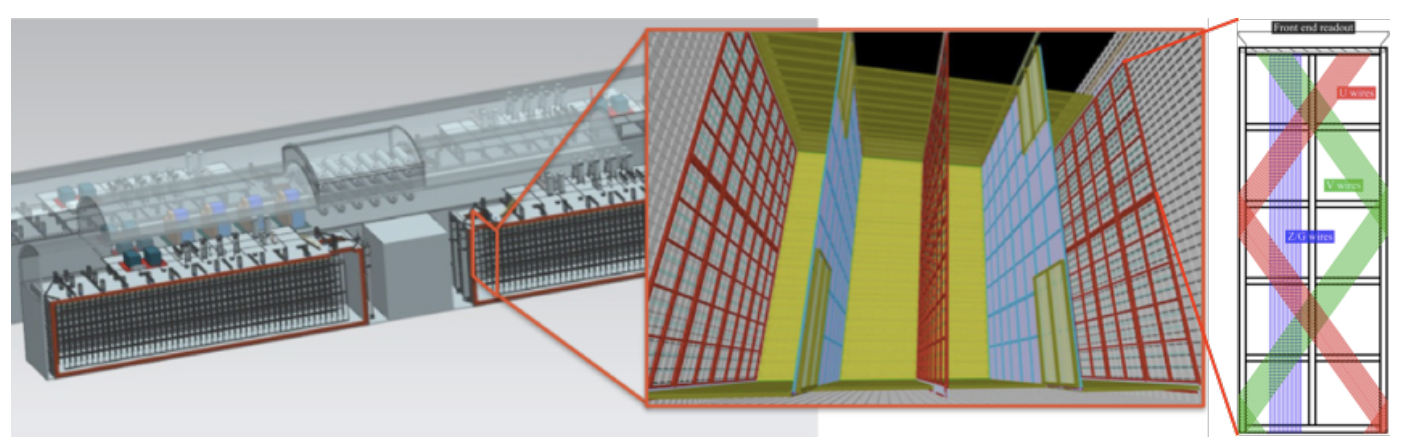

Figure 1: The DUNE single-phase detector design. The full far detector, with the four $10 \mathrm{kt}$ modules, is visible at the left of the figure and a schematic of the modules, viewed along its length (beam direction into the page), is shown at the centre. The red planes are anodes and the blue cathodes. A schematic of an Anode Plane Assembly (APA), one anode unit, is shown at the right hand side. The three planes are collection (blue) and two induction (red and green). Adapted from [2].

tific application and has taken data at Fermilab in two Phases: Phase I (December 2013 - February 2014) and Phase II (January - March 2016).

Phase I $[4,5]$ was solely a test of the cryostat concept and purification system, without a detector, to confirm the required LAr purity may be achieved and maintained in such a system. Phase II included a detector (TPC, photon detectors, external muon counters) and took data from cosmic interactions. The experiences of this run, including analysis of the data taken, is the subject of these proceedings. In both phases, an electron lifetime of $3 \mathrm{~ms}$ was achieved and held, validating the cryostat technology and the detector integrated system.

\subsection{The 35-ton Detector}

The 35-ton detector is demonstrated in Figure 2. Multiple (two) drift regions are read out by four DUNE-style APAs, with a collection plane and two planes of wrapped induction wires, instrumented with 2048 total channels and cold analogue and digital electronics. The APAs contain integrated photon detectors with SiPM readout, similar to the current DUNE far detector. An additional external triggering system is provided by a set of $\sim 100$ scintillation paddles positional around the outer cryostat walls. Finally, a set of eight optical cameras were installed inside the cryostat to search for high voltage breakdowns [6]. This design prototyped many features of the DUNE far detector such as the segmented TPC, integrated PDS, multi-drift region and cold electronics.

\subsection{Phase II Outcomes}

The run yielded 22 days of cosmic events, triggered on counter coincidences by through-going particles, taken with good LAr purity ( $\geq 3 \mathrm{~ms}$ ) and in the presence of an electric field (high voltage of $250 \mathrm{~V} / \mathrm{cm}$; half the design field). The run ended prematurely early one morning when the shearing of a pipe, part of the purification system, facilitated the introduction of air into the system, poisoning the LAr volume almost instantaneously.

The 35-ton has resulted in many favourable outcomes and a number of lessons learned. The modular TPC design has been validated and automated reconstruction has been shown to work 


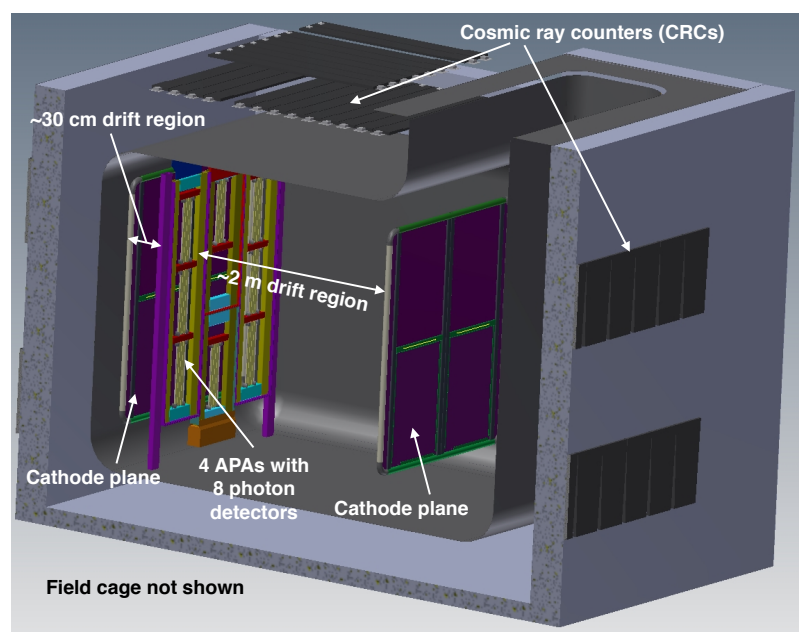

(a) Schematic of the 35-ton detector.

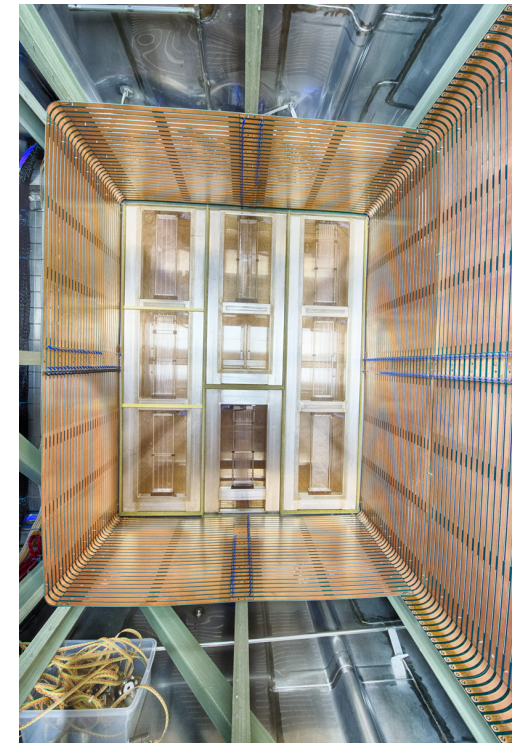

(b) Photo of the partially constructed detector.

Figure 2: The 35-ton detector

on real data, a significant step in the DUNE programme. The high voltage system, data acquisition (DAQ) and PDS were also a success but unfortunately, due to the abrupt end of data taking, were unable to be tested to the intended extent. Further complications with the TPC data, notably unexpectedly high levels of electronic noise, has resulted in data analyses focusing more on the unique aspects of the 35-ton data such as measurements of the modular TPC design, making use of the external counters for positioning and timing and understanding the performance of the photon detectors in the integrated system.

\section{35-ton Data Analyses}

\subsection{Detector-Crossing Muons}

Straight tracks which cross the detector can be used to make measurements in two ways: tracks which pass between individual drift volumes, read out by separate APAs, can be used to measure the gaps in between the APAs; tracks which pass through the APAs, read out on both sides, may be used to measure the absolute interaction time of the event. In both cases, unexpected offsets were found in the 35-ton data. In the former case, evidence for an offset in the drift direction (in addition to the expected offsets along the direction parallel to the collection wires) was found, and a method to measure both simultaneously was developed. In the latter case, a timing offset was noticed between detector sub-systems, of around $32 \mu \mathrm{s}$, which was previously unknown. Both analyses will be highly useful calibration tools for the DUNE far detector.

\subsection{Charge Deposition in TPC}

Tracks which pass through the APAs may also be utilised to study the deposition of charge 
as the track propagates. Due the grounded mesh at the centre of each APA, a strange effect has been observed; if the ionising track liberates electrons after passing the collection plane then the 'backward'-facing field between the mesh and the anode wires will result in the electrons apparently drifting backwards in time. This manifests as a large number of hits occurring at exactly the interaction time and also as an observable geometric effect in event displays and is not currently taken into account in the DUNE simulation.

\subsection{Measuring T0 from Diffusion}

As drift electrons travel from their source toward the readout wires, thermal motion results in 'diffusion' both longitudinally (in time) and transversely (between wires). The width of the induced charge from the electron packets at the readout wires is related to how far the charge has propagated; a longer drift would result in a spreading out of electrons in time and therefore a wider hit. It is therefore possible in principal to infer the start position of a track, and therefore absolute interaction time, from the width of its hits. This has been shown to work reasonably well in the 35-ton data, and significantly better in a simulated lower-noise detector. Whilst not able to give absolute event times, the technique may be used as an additional handle in matching multiple flashes to their corresponding tracks.

\subsection{Measuring Purity from Tracks}

The charge attenuated due to electronegative impurities in the LAr is parameterised as an exponential decay with constant inversely proportional to the electron lifetime. By selecting tracks with known interaction time and fitting this exponential decay, a measurement of the LAr purity can be made directly from the data. This additionally involved careful simulations of the 35-ton with a 'data-driven' noise model to correctly account for the problems with the electronics. A measurement of $4.25 \pm 0.24$ (stat.) \pm 0.28 (sys.) ms was determined, in reasonable agreement with the measurements from the dedicated purity monitors from the time the data were taken.

\subsection{Photon Detector Performance}

The PDS successfully demonstrated the APA-embedded style and development with the system and readout has been carried forward for use in ProtoDUNE-SP. Analysis combined with external counter data have demonstrated excellent timing resolution of better than $100 \mathrm{~ns}$. The characteristic length of light within LAr, determined by considering the scintillation light yield for multiple tracks at different distances from, but roughly parallel to, the APAs, was determined to be $155 \pm 28 \mathrm{~cm}$.

\section{Lessons Learned}

Many lessons have been learned alongside the positive outcomes of the 35-ton prototype. Experiences in the commissioning and running, such as how to recover from power outages and the unfortunate premature run end, will be taken forward. The current design considerations for the far detector have also been influenced by the 35-ton, such as the induction wire angle of $37^{\circ}$ (from $45^{\circ}$ in the 35 -ton) and the change to inserting the photon detector lightguides after wire wrapping rather than before. 
The greatest problem with the 35-ton data was the electronics noise in the readout and a dedicated effort following the run end has ensured that all sources of noise have been identified and understood. Two main frequency bands were determined to be due to shorts in electrical circuitry and a large correlated component from channels sharing a voltage regulator. The digitiser also had a stuck bit issue, regularly erroneously reporting particular values. The development of the electronics for DUNE is continuing using the 35-ton experience.

\section{Summary}

The 35-ton was a successful first prototype for DUNE and is already influencing the design and schedule of the DUNE far detector, along with the preparations for the next prototype, ProtoDUNESP. This will be a full-scale (APAs, CPAs, drift distance) engineering prototype in a CERN test beam and is scheduled to take data in late 2018. Many of the 35-ton analyses and experiences in detector installation, commissioning and running from the 35-ton are vital for the success of ProtoDUNE-SP and the continuation of the thriving DUNE programme.

\section{References}

[1] DUNE Collaboration, Long-Baseline Neutrino Facility (LBNF) and Deep Underground Neutrino Experiment (DUNE): The LBNF and DUNE Projects, arXiv:1601.05471 [physics.ins-det] (2016), [1601.05471].

[2] DUNE Collaboration, Long-Baseline Neutrino Facility (LBNF) and Deep Underground Neutrino Experiment (DUNE): The DUNE Detectors at LBNF, arXiv:1601.02984 [physics.ins-det] (2016), [1601.02984].

[3] D. Montanari and Others, First scientific application of the membrane cryostat technology, AIP Conf. Proc. 1573 (2014) 1664-1671.

[4] A. Hahn, M. Adamowski, D. Montanari, B. Norris, J. Reichenbacher, R. Rucinski et al., The LBNE 35 Ton Prototype Cryostat, in Proceedings, 21st Symposium on Room-Temperature Semiconductor X-ray and Gamma-ray Detectors (RTSD 2014): Seattle, WA, USA, November 8-15, 2014, p. 7431158, 2014, DOI.

[5] D. Montanari, M. Adamowski, A. Hahn, B. Norris, J. Reichenbacher, R. Rucinski et al., Performance and results of the LBNE 35 ton membrane cryostat prototype, Physics Procedia 67 (2015) 308-313.

[6] N. McConkey, N. Spooner, M. Thiesse, M. Wallbank and T. K. Warburton, Cryogenic CMOS cameras for high voltage monitoring in liquid argon, Journal of Instrumentation 12 (2017) P03014. 